\title{
Functional clustering in hippocampal cultures: relating network structure and dynamics
}

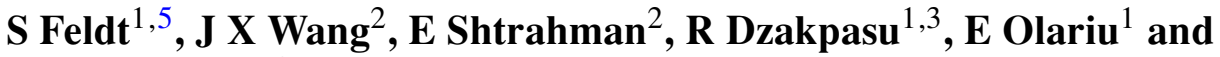 \\ M Żochowski ${ }^{1,4}$ \\ ${ }^{1}$ Department of Physics, University of Michigan, Ann Arbor, MI 48109, USA \\ 2 Applied Physics Program, University of Michigan, Ann Arbor, MI 48109, USA \\ ${ }^{3}$ Department of Physics, Georgetown University, Washington, DC 20057, USA \\ ${ }^{4}$ Biophysics Program, University of Michigan, Ann Arbor, MI 48109, USA \\ E-mail:sfeldt@uci.edu
}

Received 4 July 2010

Accepted for publication 28 September 2010

Published 26 October 2010

Online at stacks.iop.org/PhysBio/7/046004

\begin{abstract}
In this work we investigate the relationship between gross anatomic structural network properties, neuronal dynamics and the resultant functional structure in dissociated rat hippocampal cultures. Specifically, we studied cultures as they developed under two conditions: the first supporting glial cell growth (high glial group), and the second one inhibiting it (low glial group). We then compared structural network properties and the spatio-temporal activity patterns of the neurons. Differences in dynamics between the two groups could be linked to the impact of the glial network on the neuronal network as the cultures developed. We also implemented a recently developed algorithm called the functional clustering algorithm (FCA) to obtain the resulting functional network structure. We show that this new algorithm is useful for capturing changes in functional network structure as the networks evolve over time. The FCA detects changes in functional structure that are consistent with expected dynamical differences due to the impact of the glial network. Cultures in the high glial group show an increase in global synchronization as the cultures age, while those in the low glial group remain locally synchronized. We additionally use the FCA to quantify the amount of synchronization present in the cultures and show that the total level of synchronization in the high glial group is stronger than in the low glial group. These results indicate an interdependence between the glial and neuronal networks present in dissociated cultures.
\end{abstract}

\section{Introduction}

The study of networks and their role in social, technological and biological settings has recently been realized to be of great importance in understanding these complex systems. However, much work has focused on the study of the structural properties of static networks in which the nodes and edges of the network remain constant over time or are examined over a snapshot or time window during which they are effectively

5 Present address: Department of Anatomy and Neurobiology, University of California, Irvine, CA 92697, USA. static [1-5]. While this work has contributed much to our understanding of networks, it fails to recognize that many types of networks are composed of dynamic elements which are connected to form a specific function. In these dynamic networks, we can define a new type of structure called functional structure. Here connections between nodes are derived from a functional relationship between their dynamics.

The brain is a specific example of a dynamic network in which functional structure has gained a great deal of attention [6-9]. When studying the brain, one can examine the relationship between the anatomical network structure of the 
neurons and the resulting dynamics which define the functional network structure. The availability of new data from largescale brain imaging techniques such as fMRI has allowed for the large-scale studies of the relationship between structure and dynamics of different brain regions, and often one finds a correlation between the anatomical connections between brain regions and the resulting functional structure [10-13].

However, while functional connections between neurons can exist due to underlying anatomical connections, intrinsic neuronal properties and/or chemical imbalances can also alter brain dynamics [14]. For example, computational studies modeling epilepsy have shown that changing either intrinsic neuronal excitability (but not network topology) [15] or network topology (but not excitability) [16] can lead to increased network synchronization. It is therefore imperative to develop analysis and experimental methods that allow one to probe the functional structure of neuronal networks under a variety of conditions in order to determine the underlying cause of the observed functional relationships. Specifically, one would like to be able to relate differences in functional network structure to differences in anatomical network structure or changes in neuronal properties.

In order to relate functional structure to other network properties, one must first define how functional structure is deduced. Here we will focus on the use of the functional clustering algorithm (FCA) which was recently developed to define groups of similar neurons based upon statistically significant relationships between their firing events. When combined with a recently published similarity metric (average minimum distance (AMD)) which detects synchronization in discrete event data, this algorithm can detect groups of neurons which fire synchronously when applied to spike train data [17]. This allows one to record the activity of neurons in the form of spike trains and deduce functional network structure directly from the dynamics of the neurons. When combined with an experimental setup in which neuronal dynamics can be easily recorded under different conditions, this provides a powerful tool for relating functional structure with other network properties.

Dissociated cultures are a good reduced system in which to study neuronal dynamics because they maintain many properties of neuronal interactions, but also have the advantage of the relative ease of structural and pharmacological manipulation [18-22]. The gross structural properties of the culture can be observed using labeling techniques and dynamics of single neurons can be electrically recorded. They are thus a useful tool to simultaneously study how anatomical connectivity, functional structure and brain dynamics relate.

In this type of culture preparation, a single cell suspension of hippocampal cells is plated onto a culture dish. The neurons attach to the surface of the dish and start to grow processes, forming synapses with other neurons and creating a two-dimensional network of neurons. After 3-7 days, these neurons will begin to spontaneously fire action potentials, and as the cultures age, the neural activity evolves into highly synchronous activity in the form of network bursts, during which almost all neurons will fire action potentials [23, 24]. The dynamics of these network bursts have been studied to examine burst patterning [25-29], learning [20, 21, 30, 31], and with pharmacological manipulation as a model of epilepsy [32-34].

An often overlooked feature of these cultures is that they are composed of both neural and glial cells, and in fact, the neurons require the presence of glial cells to survive. While neurons do not proliferate in the culture, the glial cells do, and thus the number of glial cells in the culture will quickly multiply, creating a confluent layer of support cells for the neurons. However, it is possible to inhibit the division of these cells through the addition of chemical blockers [35] or the type of media used [36]. Different groups have studied the dynamics of the neurons in cultures grown under both types of conditions, although many studies allow the glial cells to multiply in order to provide a continuous layer of support for the neurons [24, 27, 37].

While the dynamics of the network bursts produced by the neurons in these cultures are often investigated, the impact of the glial network on the neuronal dynamics is less studied. However, recent work suggests that glial cells, and specifically astrocytes (a certain type of glial cell), can have numerous impacts on neuronal networks [38]. The presence of glial cells is known to impact the development of neurons [39], which will certainly affect their dynamics, but astrocytes have also been shown to exhibit calcium oscillations which can affect intracellular calcium dynamics in neurons [40-43]. Calcium plays multiple roles in neuronal signaling, so these calcium oscillations likely contribute to the activity of the neurons. Additionally, astrocytes have been shown to mediate calcium waves during the developmental stage (first two weeks) of dissociated cortical networks, and the presence of these waves influences the dynamics and maturation of the culture [44]. Recent work has also shown that astrocytes modulate slow oscillations in cortical networks through the regulation of synaptic receptors [45]. We therefore expect that changes in the glia network should impact the dynamics of the neuronal network in developing cultures.

In this paper, we are interested in studying differences in the functional structure derived from the dynamics of the neuronal network in the presence of either a low or high density of glial cells. We investigate dissociated hippocampal cultures during the developmental stage and examine the underlying anatomical network structure of both neurons and glial cells and how these networks interact to influence the resulting functional network structure. The cultures are grown on multi-electrode arrays (MEAs) which allow for the recording of individual action potentials from cells near the electrodes. We analyze the formation of spatio-temporal activity patterns using a recently published clustering technique (FCA) to determine the resulting functional network structure [17] and show that this algorithm is useful for capturing changes in functional network structure in dynamic networks which evolve over time. We then relate this evolving functional structure to gross anatomical network properties obtained through immunolabeling and DiI staining.

The paper is organized as follows. Section 2 describes the experimental methods and analysis. The results are presented in sections 3 and 4 . Section 3 describes the anatomical 
structure and dynamics of the cultures. In section 4, we focus on the resulting functional network structure and how this structure changes over time and between groups. The relationship between all three areas is discussed in section 5 , where we further describe how the structure of the glial and neuronal networks influences the dynamics and resulting functional structure of the neuronal network.

\section{Experimental methods and analysis}

\subsection{Cell culture preparation}

Dissociated cell cultures were prepared from neurons (and glia) obtained from the hippocampus of P1 Wistar rats using a protocol modified from [21]. Briefly, hippocampi were first chemically digested in a trypsin solution followed by mechanical titration with a flamed pasteur pipette. Cells were centrifuged and re-suspended in Neurobasal-A medium supplemented with B-27, 5\% heat activated horse serum, $0.5 \mathrm{mM}$ L-glutamine, $0.5 \mathrm{mM}$ pen-strep and $10 \mathrm{mM}$ HEPES. The cell density was adjusted by the addition of media such that the density upon plating would be $\sim 1400$ cells $\mathrm{mm}^{-2}$.

The cell suspension was plated on MEAs (Multi Channel Systems, Reutlingen, Germany) which had previously been coated with $0.05 \%$ poly-ethylene-imine in borate buffer followed by the $20 \mu \mathrm{g} \mathrm{mL} \mathrm{mL}^{-1}$ laminin solution in media. Cultures were maintained in a humidified incubator with a $95 \% \mathrm{O}_{2} / 5 \% \mathrm{CO}_{2}$ saturated atmosphere at $37{ }^{\circ} \mathrm{C}$.

Between 24 and 36 hours after plating, cultures were split into high and low glial groups, HGG and LGG, respectively. Neurobasal-A media supplemented with horse serum as described above was added to cultures in the HGG to allow for the proliferation of glial cells, while the media of the LGG was replaced with Neurobasal-A media that had not been supplemented with horse serum. The sera-free media environment keeps the number of glia cells in these cultures constant by inhibiting their division. Following this, half of the media was replaced with the appropriate fresh media once in a week.

\subsection{MEA recordings}

Cultures were recorded at 8,11 and 13 days in vitro (DIV) for $N=5$ cultures in the HGG and $N=4$ cultures in the LGG. For recordings, media was replaced with a recording buffer to maintain the $\mathrm{pH}$ of the culture. Cultures were recorded at $25 \mathrm{kHz}$ using a multi-channel systems data acquisition card and MC-Rack software. During the recordings, cultures were maintained at $37{ }^{\circ} \mathrm{C}$ and each recording lasted $5 \mathrm{~min}$.

\subsection{Cell fixation and staining}

Cultures used in staining studies were grown on culture dishes following the same protocol as those used for recordings. The cultures were fixed using $4 \%$ paraformaldehyde in phosphate buffered saline (PBS) at either 8, 11 or 13 DIV to correspond to the days of recordings. DiI crystals were dissolved to a saturated solution in cold liver oil and micro-droplets of the solution were placed on neuronal cell bodies, allowing the dye to dissolve through the cell. After 5 days, the neurons were imaged using an Olympus IX71 microscope.

After DiI staining and imaging, cultures were immunolabeled for synapses by standard immunocytochemical techniques. Samples were blocked with the blocking solution, followed first by incubation with a monoclonal anti-synaptophysin primary antibody, and then by a fluorescein-conjugated secondary antibody. Cultures were then imaged on a Zeiss LSM 510-META laser-scanning confocal microscope with a $63 \times$ objective. After imaging, samples were labeled for glial cells using an anti-glial fibrillary acidic protein (GFAP) primary antibody followed by an AMCA-conjugated secondary antibody. Cultures were then imaged on a Deltavision-RT fluorescent microscope with a $10 \times$ objective.

\subsection{Process length/form}

Sholl analysis [46] was performed on the arborization of imaged neurons by counting the number of process crossings with concentric circles of increasing radii centered on the soma. The median crossing distance, total number of crossings and longest process length were then computed from the distribution of process crossings. For cultures in the HGG, this analysis was done on $N=9,11$ and 14 randomly chosen neurons for DIV 8,11 and 13 respectively and on $N=10,12$ and 13 neurons for DIV 8, 11 and 13 in the LGG, respectively.

\subsection{Synaptic density}

DiI-synaptophysin labeled images were analyzed using ImageJ software for synaptic density along neuronal processes. Images were normalized for contrast and each channel was thresholded to distinguish the signal from background. The density of synapses was calculated by dividing the number of co-localized areas of synapse and process by the total area encompassed by processes, resulting in the number of synapses per unit area of processes stained by DiI.

\subsection{Glial cell density}

GFAP labeled images were analyzed using ImageJ software to determine the glial cell density. Images were thresholded to separate the signal from background and the percentage of the image covered in glial cells, $P$, was measured. In order to compare different culture conditions, we compute the normalized difference of $P$ between the HGGs and the LGGs denoted as $P_{\mathrm{H}}$ and $P_{\mathrm{L}}$, respectively:

$$
D=\frac{\left[P_{\mathrm{H}}-P_{\mathrm{L}}\right]}{\left[P_{\mathrm{H}}+P_{\mathrm{L}}\right]} .
$$

\subsection{Spike detection}

The local field potential recorded from each electrode was assessed for spiking activity and active channels were selected for spike detection. Signals were first filtered through a highpass Butterworth filter at $250 \mathrm{~Hz}$. Spike detection was done 
using a thresholding method, using five standard deviations of the baseline noise as the threshold value. Since it is difficult to reliably separate individual neuronal spikes in these types of bursting recordings [25], no attempt was made to distinguish between single neurons recorded by the same electrode. However, due to the moderate plating density, we expect that each electrode will record the activity of only a single or a few neurons.

\subsection{Cross-correlation}

Spike trains from each active electrode were first convolved with a Gaussian kernel with a standard deviation of $1 \mathrm{~ms}$ and the signal was demeaned (the mean value of the signal was subtracted). The cross-correlation is then given by

$$
\hat{C}\left(S_{i}, S_{j}\right)=\frac{C\left(S_{i}, S_{j}\right)}{\sqrt{C\left(S_{i}, S_{i}\right) \cdot C\left(S_{j}, S_{j}\right)}},
$$

where $C$ is the linear cross-correlation function:

$$
C\left(S_{i}, S_{j}\right)=\int_{-\infty}^{\infty} S_{i}(t) S_{j}(t) \mathrm{d} t
$$

The average value of cross-correlation was then computed as a function of distance, where the distance was binned in intervals of $100 \mu \mathrm{m}$ and averaged over cultures within each group.

\section{Results: anatomical structure and dynamics}

We analyze structural and functional connectivity in dissociated hippocampal cultures grown under conditions which allow for the proliferation of glial cells (HGG) or inhibit the division of glial cells (LGG). Cultures were grown either on culture dishes and used for immunolabeling/staining studies (figures 1 $(a)$ and $(b)$ ) or on MEAs for the recording of neuronal dynamics (figures $1(c)$ and $(d)$ ). Here, we present results describing the properties of the anatomical network structure during the developmental stage of the cultures and describe the neuronal dynamics which correspond to these underlying structural networks.

\subsection{Anatomical network structure}

We first examined the anatomic structural properties of the cultures. Although it is not feasible to determine the exact connectivity structure of the cultures (despite the reduction of the system, the resulting network is still quite complex), we are capable of studying gross properties of the network structure which could lead to differences in dynamics and functional structure. As discussed previously, we control the proliferation of the glial cells such that, over time, we obtain a HGG and a LGG. Although the neurons do not proliferate over time, they do grow processes and synapses, changing the neuronal network structure as the cultures age.

In order to quantify the differences in the glial network between the two culture groups, we labeled astrocytes as previously described and computed the normalized difference (section 2.6) between the percentage of area covered by astrocytes for cultures in the HGG and LGG. This quantity (a)
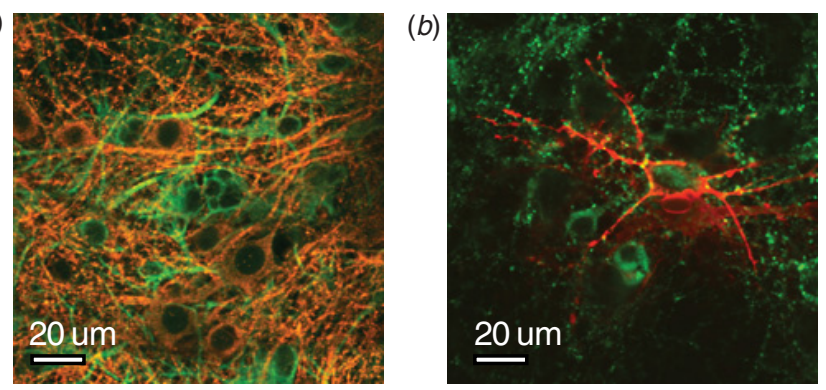

(c)
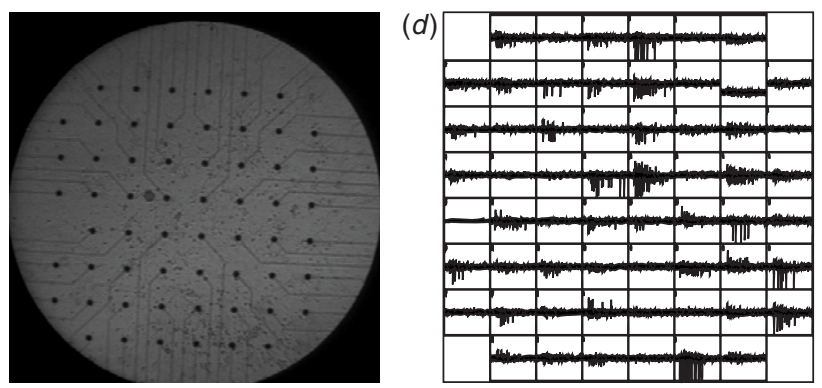

Figure 1. (a) Immunocytochemical labeling of neurons and astrocytes showing morphology. Neurons are immunolabeled for beta-III tubulin (red), while astrocytes are immunolabeled for GFAP (green). (b) Example of an isolated neuron stained with DiI (red). Synapses are immunolabeled for synaptophysin (green). (c) Example of a culture grown on an MEA showing the spatial layout of the electrodes (black dots) The distance between electrodes is $200 \mu \mathrm{m}$. (d) Example of recorded activity from a culture displaying bursting dynamics. The spatial layout of the activity traces corresponds to the spatial layout of the electrodes shown in $(c)$. Each window represents a $500 \mathrm{~ms}$ time window during which the synchronous firing of neurons can be seen.

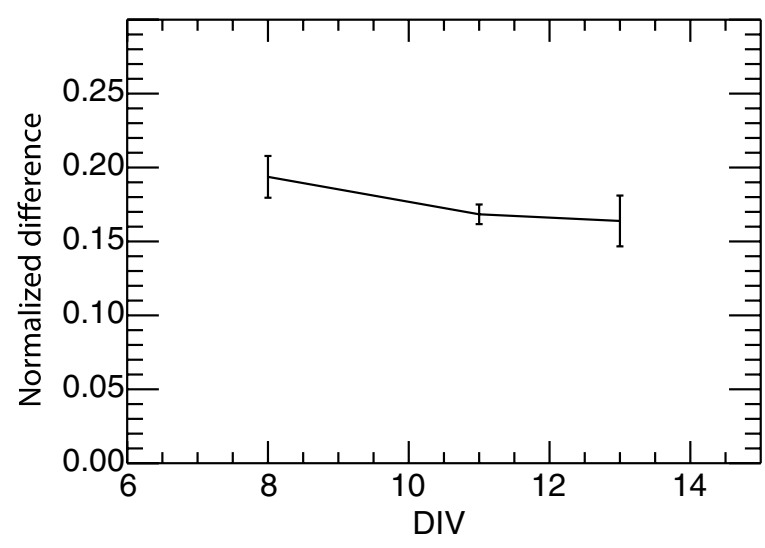

Figure 2. The normalized difference calculated between the percentage of coverage of glial cells in the HGGs and LGGs (equation (1)). This measure remains approximately constant over time due to the fact that the glial cells do not multiply in the LGG and have already covered the culture dish by 8 DIV in the HGG.

is plotted as a function of DIV in figure 2. The measure shows that, as expected, the area covered by astrocytes is higher for the HGG, and that the ratio of coverage between the culture groups remains fairly constant over time (due to the fact that astrocytes do not proliferate in the LGG and have already formed a confluent layer by 8 DIV in the HGG). 


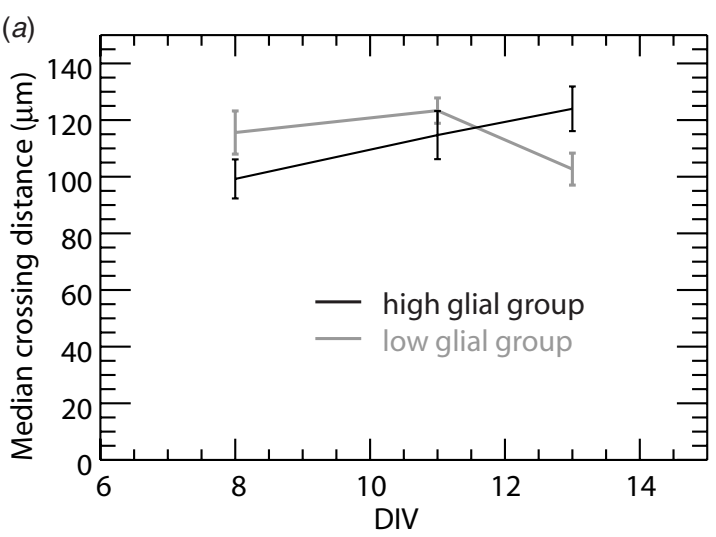

$(c)$

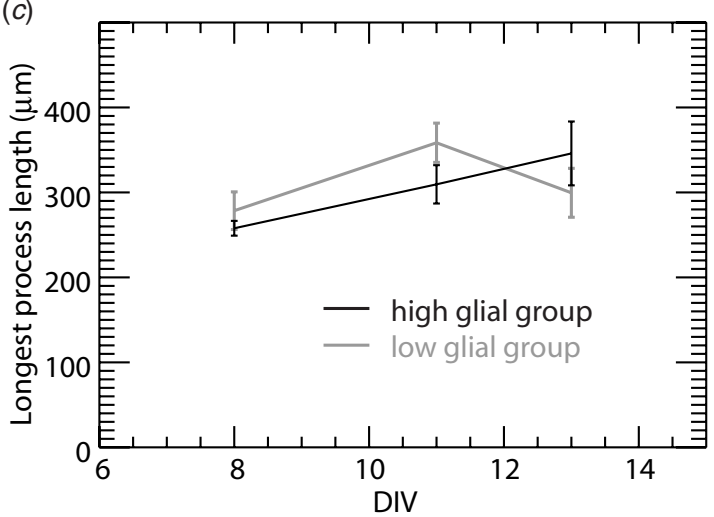

(b)

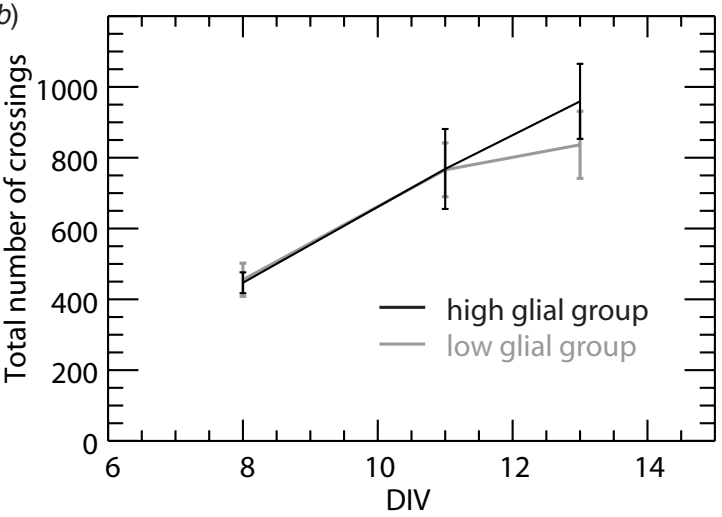

(d)

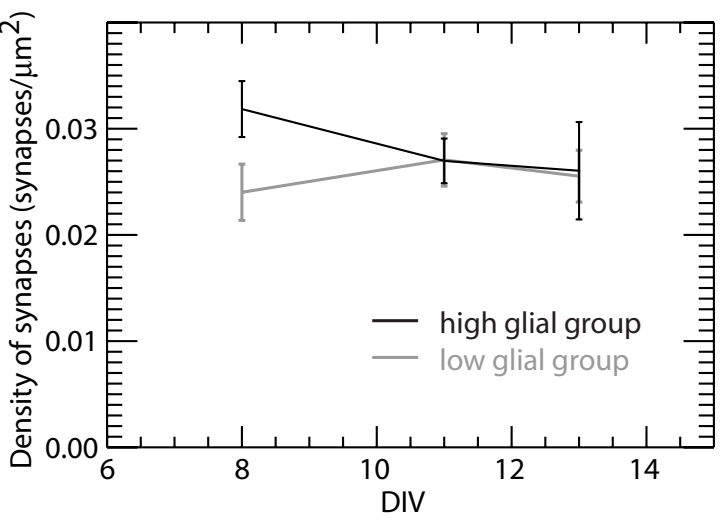

Figure 3. Neuronal process morphology and synapses. (a)-(c) Properties of the distribution derived by a Sholl analysis conducted on DiI labeled neurons (see section 2.4). (a) Median crossing radius as a function of DIV. (b) Total number of crossings. (c) Longest process length. $(d)$ Density of synapses co-localized with DiI stained neuronal process, as a function of DIV.

We then studied the structure of the neuronal network for the two culture groups using a Sholl analysis described in section 2.4. From the distribution of the number of process crossings as a function of radii, we computed the median crossing distance, the total number of crossings and the longest process length for each neuron. These results are presented as a function of DIV in figures 3(a)-(c) for each culture group. We see changes as the cultures age and in certain cases between groups for these measures. The median crossing distance $(a)$ and the longest process length $(c)$ increase as a function of time for neurons in the HGG, but both measures show a slight decrease for 13 DIV cultures in the LGG. This represents a re-organizing of the distribution over time since the total number of crossings increases as the cultures age as depicted in figure $3(b)$. Neurons in the LGG are growing more local arborizations, as compared to those in the HGG whose processes continuously increase in length over time. However, it should be noted that the density of synapses along these processes is similar over time between the two culture groups, although it is slightly higher for neurons in the HGG initially (figure $3(d)$ ).

Together, these labeling results indicate that neurons in the LGG are involved in more local signaling, while those in the HGG are developing more long-range connections.

\subsection{Dynamics}

We studied the dynamics of the cultures as a function of age to determine the effects of the changing glial network and growth of neuronal processes. Cultures were recorded from 8, 11 and $13 \mathrm{DIV}$ as described in section 2. Spike detection was done on the recorded signals and the resulting spike activity was analyzed as follows.

The visual observation of the recording sessions shows that cultures in the HGG tend to persistently fire in network bursts with short silences in between bursts, while those in the LGG tend to fire in a single long burst with longer quiescent periods between bursting events. These quiescent periods grow in length over time. In order to show these differences in neuronal dynamics as the two culture groups evolve, we present raster plots of bursting activity at successively smaller time scales in figure 4. In figure 4(a) we show $100 \mathrm{~s}$ of bursting activity for cultures in the HGG (two columns on the left) and LGG (two columns on the right) at 8 and 13 DIV. This time scale allows one to examine the bursting activity at large-burst time scales. We look at progressively smaller (10 s and $1 \mathrm{~s}$ ) windows in figures $4(b)-(c)$. Here, raster plots are shown for the time window enclosed by the shaded box in the above panel. One can see that the larger bursts depicted at the $100 \mathrm{~s}$ time scale are actually composed of a much finer structure of smaller bursting behavior. As the cultures develop, 

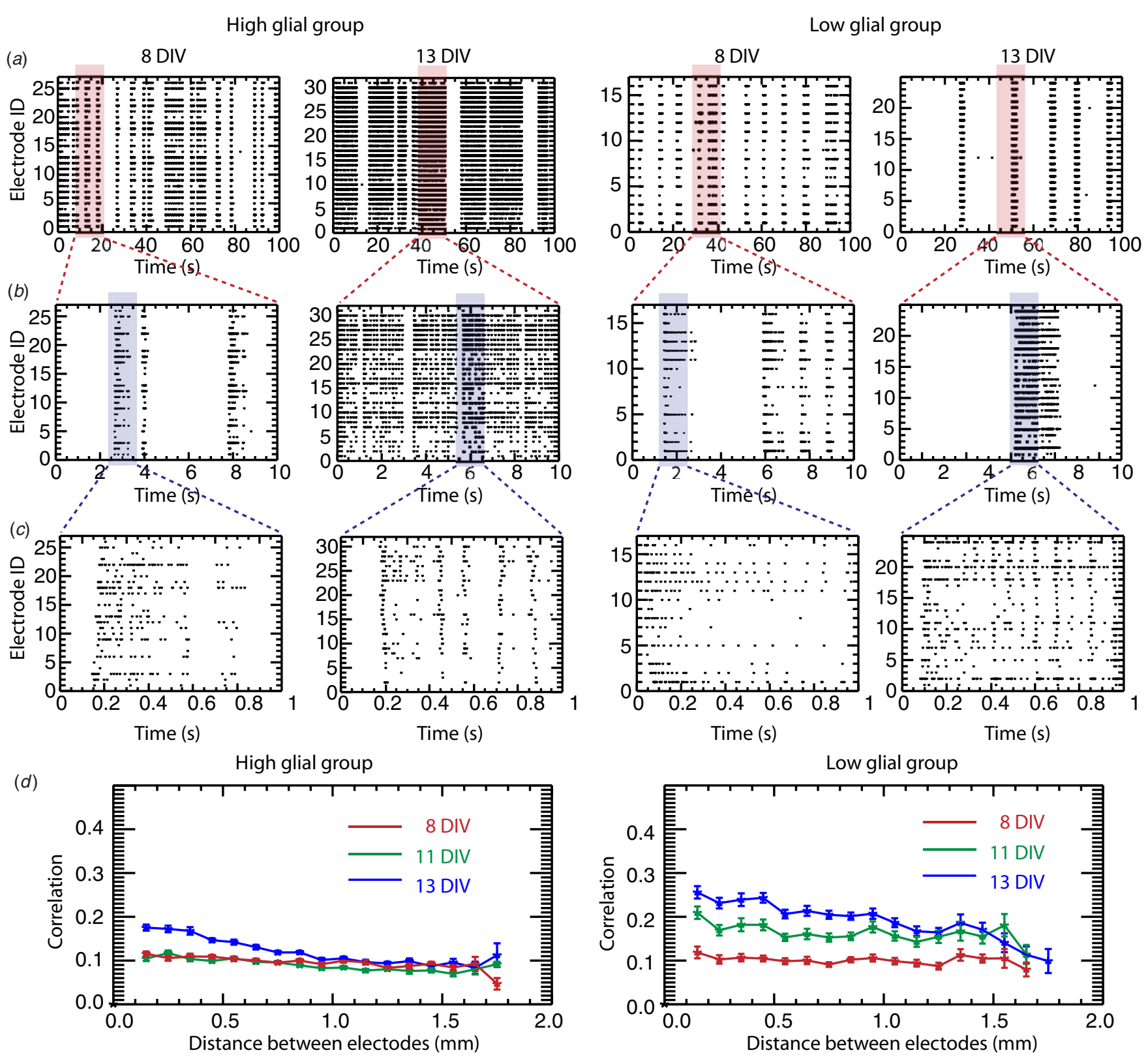

Figure 4. Examples of typical raster plots from a culture in the HGG and the LGG at 8 and 13 DIV. (a) Shown at a time scale of $100 \mathrm{~s}$ to visualize large-scale bursting behavior. (b) Activity shown at the $10 \mathrm{~s}$ time scale for data enclosed in the red box in the above plots. $(c)$ Short time scale bursting activity shown for $1 \mathrm{~s}$ of data from the area enclosed in the blue box in the above plots. $(d)$ Cross-correlation as a function of the distance between electrodes for cultures in the HGG (left) and the LGG (right). Spikes were convolved with a Gaussian with a standard deviation of $1 \mathrm{~ms}$ to detect small time scale correlations within firing patterns.

we see changes in the structure of this behavior at each time scale. At 8 DIV, both culture groups fire with a somewhat similar structure at each time scale. However, by 13 DIV, we see that differences in the bursting behavior of the have become apparent at each time scale. While the HGG shows long periods of large-scale bursting at the $100 \mathrm{~s}$ time scale, the LGG bursts in shorter burst events with long silent periods. If we examine these bursts at the $10 \mathrm{~s}$ scale, we can see that the longer bursts of the HGG are actually composed of shorter events of bursting separated by short silences, while the LGG fires in a burst of continuous activity. If we again examine these bursts at the $1 \mathrm{~s}$ time scale, it becomes apparent that the bursting of the HGG contains a bursting structure at an even shorter time scale. While some of this bursting structure is evident at 13 DIV in the LGG, it is clear that the firing here is more continuous during the length of the large-scale burst and there is less overall short-scale bursting behavior.

As expected, examining the correlation of neuronal activity as a function of the distance between electrodes shows no relationship at large time scales as the large-scale bursting behavior is global (with nearly all active electrodes showing activity during the burst) and possibly driven by calcium oscillations in the glial network [44]. However, at shorter time scales, the relationship between electrode distance and neuronal firing is less clear. In figure $4(d)$ we show the average cross-correlation between spiking activity for cultures at each 
stage of development plotted as a function of the distance between electrodes. For this calculation, neuronal spikes convolved with a Gaussian with a standard deviation of $1 \mathrm{~ms}$ (see section 2.8). While there is no relationship between distance and correlation for 8 and 11 DIV cultures, we see that in both culture groups, at 13 DIV, there is an increase in the correlation between electrodes which are spatially closer to each other. This indicates that changes in the networks of both culture groups allow the neuronal network structure to more highly influence the overall dynamics at the shorter time scales. The fact that the overall correlation is higher for neurons in the LGG results from an increased density of neuronal firing during bursts as discussed below. This suggests that measures which require discrete binning such as crosscorrelation (despite being normalized for firing frequency) should be used with caution when examining the dynamics of spiking activity with structure at very short time scales as exhibited here, as the binning or convolution can blur detailed patterning present at these short time scales.

The changes in the neuronal network are also apparent when examining the number of active electrode channels as a function of DIV. An active electrode was defined as a channel from which reliable spiking activity could be detected using the thresholding method. It should be noted that in order to record the activity from a neuron, the neuron must lie very near to the electrode and be well attached to the surface of the dish. As the network ages, some neurons die and others begin to fire, meaning that the active electrodes can change over time. However, one expects the overall number of active electrodes to increase over time as the neuronal processes grow, allowing for more neurons to be recruited into spontaneous activity as the network becomes more highly connected. The number of active electrodes is plotted for both the HGGs and the LGGs in figure 5(a). In both groups this number increases over time, yet is consistently higher for the HGG. This indicates that the higher number of glial cells provides additional support for the neuronal network, allowing for the recruitment of more spatially separated sites into the bursting activity.

In figure 5(b), we plot the average number of spikes per active electrode as a function of time. Cultures from each group exhibit nearly the same number of spikes which tells us that the differences seen in the bursting structure between the two culture groups represent differences in network organization and not simply a lack of activity in one culture group. Thus, the firing rate of neurons during bursts in the LGG at 13 DIV is higher than those in the HGG as cultures in the LGG display large quiescent periods with no or minimal spiking.

In the following sections we will present a methodology to quantify these differences in dynamics based on functional network structure. In order to account for the observed fine scale structure of the bursting dynamics, we present a distance metric designed for discrete event data and use this measure along with a FCA to quantify spatial and temporal interactions between electrode sites.
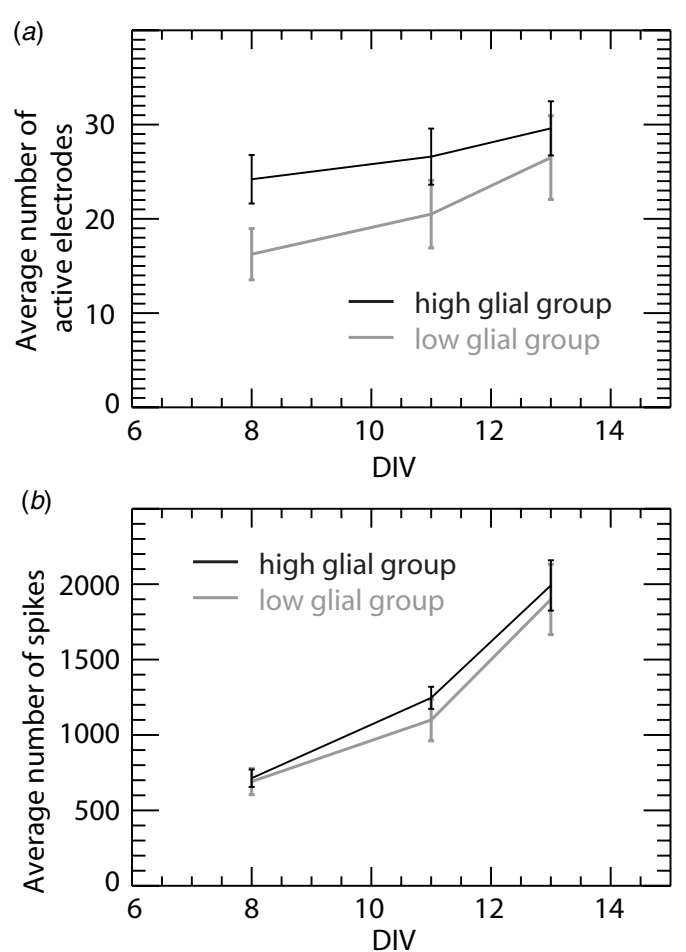

Figure 5. (a) Number of active electrodes as a function of DIV for both HGGs and the LGGs. While this number grows over time for both groups, it remains smaller for cultures in the LGG indicating that the glial network seems to influence the recruitment of sites in the neuronal network. (b) Average number of spikes per active electrode as a function of DIV during a 5 min recording. This number is similar and grows for both culture groups as the cultures become more active over time, corresponding to changes in the neuronal networks.

\section{Results: functional network structure}

\subsection{Determination of functional clusters}

Functional clustering was determined from the obtained spike train data using a clustering method recently developed in our laboratory called the FCA [17]. Advantages of using this algorithm include that the clustering is determined directly from the dynamics of the recorded neurons through a comparison to surrogate data, meaning that clustering is based on statistically significant similarities between firing patterns. The use of statistical significance to determine clustering also means that the algorithm has a natural stopping point and no a priori knowledge of the number of functional groupings is required.

This algorithm incorporates the use of a similarity metric and for the data in this paper, we use the AMD which is designed to detect co-firing events in spike train data. This new similarity metric was designed for use with the FCA and recently published in [17]. When applied to data from the previously described cultures, the AMD detects the amount of synchronization between bursting events. Since this measure is used in combination with the FCA, the result is that the clustering of spike trains is based on the amount of synchronization present between bursting events and 
(a)

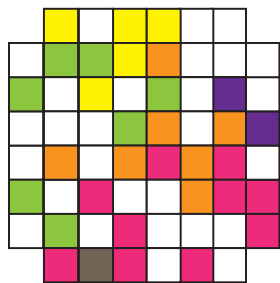

8 DIV
High glial group

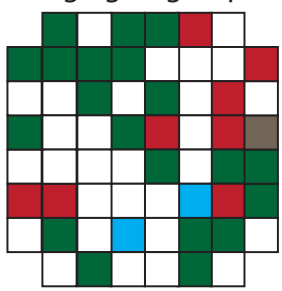

11 DIV

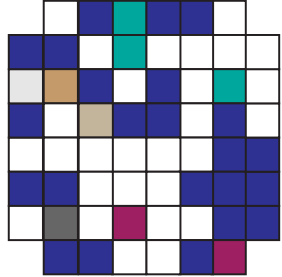

13 DIV

(b)

High glial group 8 DIV

High glial group 13 DIV

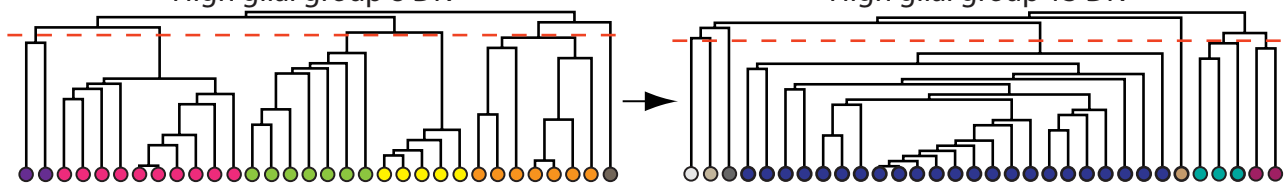

(c)

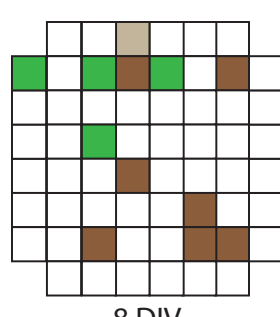

8 DIV

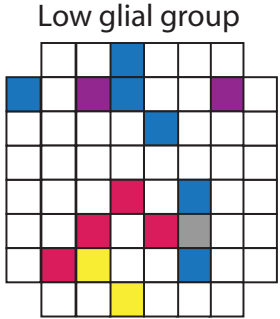

11 DIV

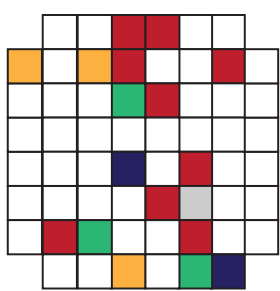

13 DIV

(d)

Low glial group 8 DIV

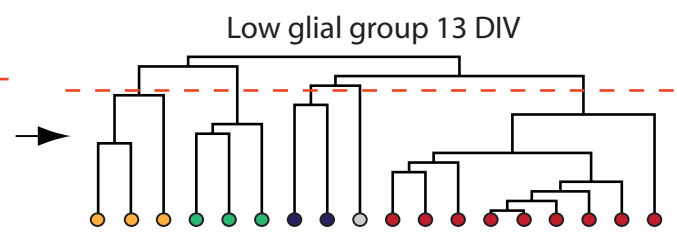

Figure 6. Examples of functional groupings obtained from the application of the FCA to culture data. (a), (c) Spatial representation of functional clusters. Colored squares indicate active electrodes and squares of the same color belong to the same functional group. (a) HGG. (c) LGG. The clustering becomes increasingly global over time for the high glial culture, while the clustering of the low glial culture becomes increasingly fragmented. $(b),(d)$ Examples of the dendrogram corresponding to the spatial maps in $(a)$ and $(c)$ at 8 DIV and 13 DIV. The dashed red line denotes the clustering cutoff.

spike trains (electrodes) whose firing patterns which show statistically significant synchronization are grouped together.

Because the algorithm directly utilizes the dynamics of the network to deduce statistically significant functional network clusters, it is ideal for detecting changes in functional network structure in networks whose dynamical properties evolve over time. By using the FCA to determine functional structure in our cultures as a function of DIV, we are able to detect changes in functional network structure over time and quantify differences between the culture groups as the cultures develop. We briefly describe the algorithm below, and for a complete description of the algorithm, please refer to [17].

The FCA can be summarized in the following five steps.

(1) Choose a similarity metric and create a matrix of pairwise similarity values between all spike trains.

(2) Use surrogate data sets (see below) to calculate 95\% confidence intervals for each pairwise similarity. Use this to determine the level of statistical significance for each pairwise relationship.
(3) Choose the pair of trains with the highest significance and group these trains together, recording the significance between the trains. When grouping the two spike trains, create a new train representing the joint activity by merging the spikes into a single train.

(4) Remove the trains which were joined from the data set, and recalculate the similarity matrix for the new set of trains. Create new surrogate data sets, and re-calculate the pairwise statistical significance.

(5) Repeat the joining steps (3) and (4), recording the statistical significance used in each step of the algorithm until no pairwise similarity is statistically significant, indicating that the next joining step is not statistically meaningful. At this point, determine the resultant functional groupings by observing which spike trains have been combined during the algorithm. The results of the clustering algorithm are depicted using a dendrogram where the dashed line denotes the cutoff point of the algorithm as in figure 6. 
In order to assess similarities between firing patterns, one must first choose a similarity metric (step 1). Here, we used the AMD which is a new measure designed to detect synchronous events in discrete event data [17], and was previously published by the authors for use with the FCA to detect clusters of synchronous neurons. To compute the AMD between two spike trains $S_{i}$ and $S_{j}$, we calculate the distance $\Delta t_{k}^{i}$ from each firing event in $S_{i}$ to the closest firing event in $S_{j}$. We then define

$$
D_{i j / j i}=\frac{1}{N_{i / j}} \sum_{k} \Delta t_{k}^{i / j},
$$

where $N_{i / j}$ is the total number of spikes in $S_{i}$ or $S_{j}$, respectively. Finally, we define the AMD to be

$$
\Theta_{i j}=\frac{D_{i j}+D_{j i}}{2}
$$

Surrogate data sets used in the calculation of significance were created through the addition of jitter to spikes (also known as dithering or teetering) [47]. The jitter is drawn from a uniform distribution over a given window. Here, we used a jitter window of $70 \mathrm{~ms}$, centered on each spike. This time scale allows us to examine synchronization at the level of network bursts that occur at short time scales within the cultures.

To assess the level of statistical significance, we used 10000 surrogate data sets to create the cumulative distribution functions (CDF) of AMD values and determine 95\% pairwise significance levels. The scaled significance (figure 8) is measured in units defined as the distance from the midpoint of the CDF to the $95 \%$ significance cutoff. Thus, a scaled significance greater or equal to 1 is deemed to be statistically significant, while values below 1 are not.

The FCA was applied to the spike train data recorded from cultures in both groups at 8, 11 and 13 DIV. In order to keep the total number of spikes used in the algorithm below 50000 , a 3 min window of data was used, with the exception of one 13 DIV culture in the LGG for which a 1 min time window was used.

We would like to emphasize that the FCA was specifically chosen for this study due to its ability to directly detect statistically significant functional groupings from the dynamics of neurons. Additionally, this algorithm (unlike many other clustering algorithms) did not require a prior knowledge of the number of functional groupings which we expected to change as the networks evolved over time.

\subsection{Differences in functional structure}

Since we observe difference in dynamics as a function of DIV as well as between culture groups, it is interesting to ask how these differences will be embodied in the functional structure of the network. To study the functional groupings of active sites, we implemented the FCA as described above.

We first explore the functional clustering as a function of time for cultures within each group. In figures $6(a)$ and (c), we show examples of the spatial layout of the functional groupings over time for the two culture groups. In this figure, each square represents the spatial location of an electrode on the culture. The spatial layout of the squares here corresponds

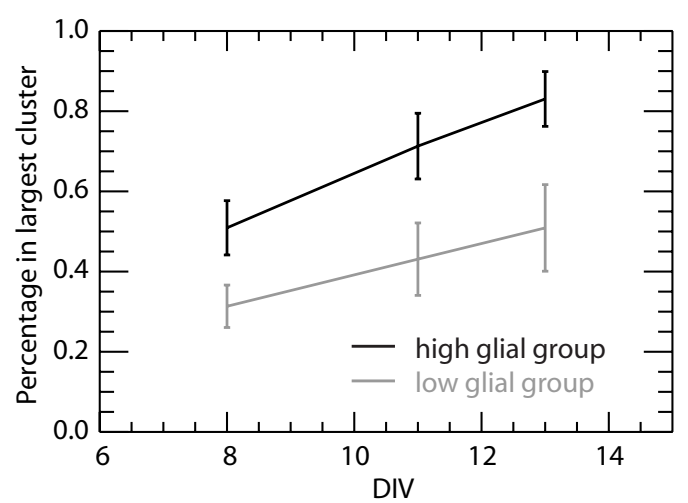

Figure 7. Percentage of electrodes participating in the largest functional cluster as a function of DIV. The percentage increases over time for the HGG indicating the spread of global synchronization. Although we also see an increase in this number for cultures in the LGG, the percentage remains smaller, as these groupings remain fragmented.

to the spatial layout of the MEAs as seen in figure 1. Colored squares represent active electrodes, and squares of the same color belong to the same functional group. The dendrograms corresponding to the spatial clustering shown in the plots of figure $(a)$ and $(c)$ at 8 and 13 DIV are shown in figures $6(b)$ and $(d)$ respectively. The red dashed line denotes the clustering cutoff as described previously. For the case of the HGG, we see that, initially, the culture contains multiple groups that largely represent local spatial regions of the MEA. This indicates that only local groups of neurons are involved in synchronous activity during network bursts. However, as the culture ages, the synchronization becomes increasingly global, and more neurons over larger spatial distances are recruited to the largest cluster. To quantify this effect, we plotted the percentage of electrodes that participate in the largest cluster as a function of DIV in figure 7.

Interestingly, we do not see this increase in cluster size in the case of cultures from the LGG, as the clustering instead remains spatially fragmented over time as seen in figure 6 . This fragmentation corresponds to the lack of change over time in the percentage of electrodes that participate in the largest cluster seen in figure 7.

Finally, we were interested in quantifying the level of synchronization present in the detected functional groupings. This was done through the examination of the scaled significance used in the joining steps of the FCA (please refer to section 4.1). An example of the scaled significance and the significant clustering steps for a 11 DIV culture is shown in figure $8(a)$. Note that a higher scaled significance indicates a tighter relationship between spikes of electrodes. We then calculated the average value of the scaled significance for the statistically significant steps for each culture. In figure $8(b)$ we show the average scaled significance used in the significant clustering steps as a function of DIV for both the HGGs and LGGs. The average scaled significance is greater for the HGG on each recording day, indicating that the high glial cultures show a tighter relationship between firing events during the bursts. However, the average scaled significance grows over 
(a)

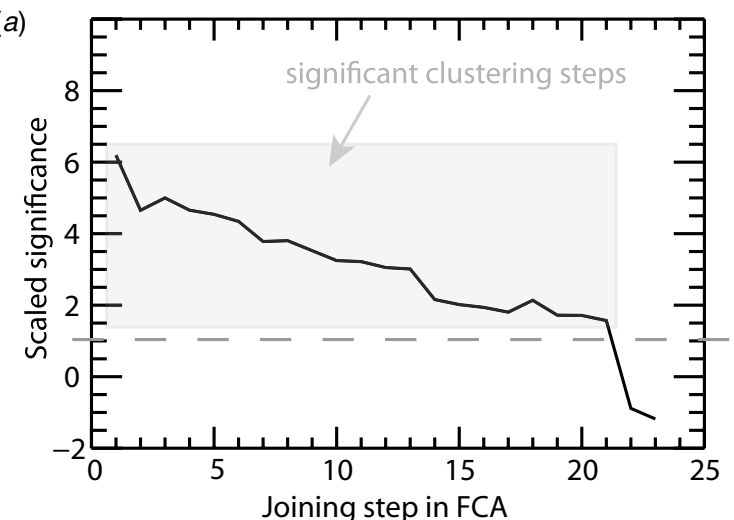

(b)

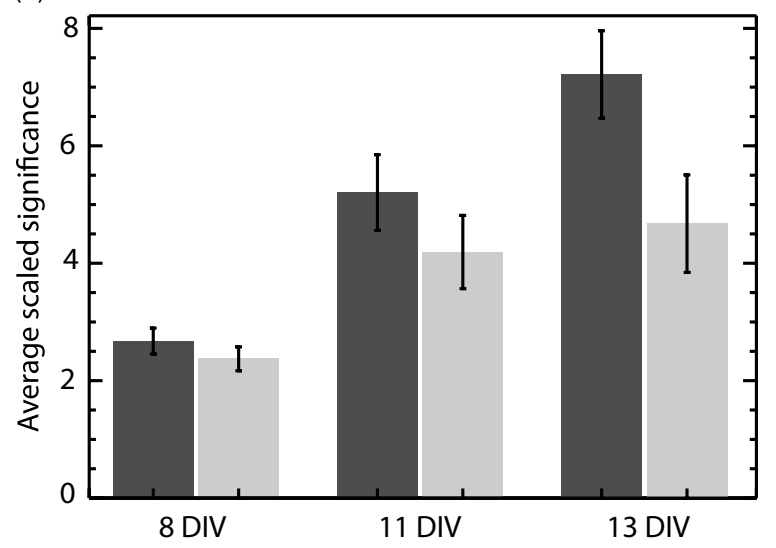

Figure 8. (a) Example of the scaled significance used in each step of the FCA for an 11 DIV culture from the HGG. The dashed line denotes the clustering cutoff, and steps above this line (enclosed in the gray box) are statistically significant. (b) The average scaled significance used in the significant clustering steps. This value was averaged over cultures from the HGG and LGG and calculated as a function of DIV. The significance increases as a function of time, indicating an increase in the synchronization of spiking within network bursts. The high glial cultures also show increased significance (synchronization) compared to the low glial cultures.

time for cultures from both groups, indicating that the firing becomes more synchronous as all cultures age.

\section{Discussion}

While the previous work has studied either structural and neuronal properties of cultured networks or analyzed the dynamics of these cultures, there is little work which attempts to link changes in neuronal network properties to the resulting dynamics and functional interactions. The work that has been done is focused on the effects of different substrates used to coat the dishes [48], on patterned plating of cultures $[26,49]$, or the excitatory versus inhibitory action of GABA as the cultures develop [37]. Here, we studied cultures grown with either a high density of glial cells or a low density of glial cells in order to relate the influence of the glial network on neuronal dynamics as a function of time (and therefore increasing density of glial cells). Additionally, we used a recently developed clustering algorithm (FCA) [17] to detect differences in functional network structure between the two groups as the cultures developed over time. It is known that the presence of glial cells shapes neuronal development [39], and Sholl analysis on the arborization of neuronal processes revealed that cultures in the HGG contained neurons whose processes grew globally over time, while cultures in the LGG grew increasingly local processes. The study of synchronization in coupled oscillators has shown that the addition of global connections (such as in a small-world network) leads to greater synchronization [50-52]. Additionally, astrocytes have been shown to mediate spontaneous calcium waves which correspond to the presence of synchronized activity in dissociated cortical cultures [44]. The presence of glial cells can also influence intrinsic neuronal properties, and astrocytes have been shown to be responsible for the modulation of slow oscillations in in vivo cortical networks through the regulation of synaptic receptors [45]. It is therefore reasonable to conclude that the observed differences in neuronal dynamics between the HGGs and LGGs are linked to the influence of the glial network on the neuronal network.

Both culture groups displayed synchronized activity in the form of network bursts, but the specific form of these bursts varied between the two groups. The high glial cultures had more active electrodes; however, the total number of spikes per electrode was similar between the two groups for each recording session. As shown in previous studies [24], the cultures became increasingly active over time as seen in the rise of active electrodes and total spikes per electrode. However, the cultures from the HGG displayed more structured bursting activity at short time scales. Cultures from the LGG displayed spiking patterns that evolved to periods of large-scale bursting with long periods of silence in between bursts, corresponding to an increase in the spiking frequency during bursts (but lack of short time scale burst structure).

The changes in neuronal dynamics over time led to a difference in functional classifications which we were able to detect through the application of our recently developed clustering algorithm. We applied the FCA to detect functional clusters and examined the spatial aspect of the resultant clusters. Cultures from the HGG initially showed the formation of local clustering which became more global over time as more electrodes participated in the largest cluster and the cluster encompassed a larger spatial area. However, cultures in the LGG showed a different behavior as the groupings remained spatially fragmented over time and fewer electrodes were included in the largest cluster. These functional differences indicate that the cultures from the HGG display bursting events at short time scales in which many neurons display synchronous spiking activity, while short time scale bursting events are consistently composed of smaller groups of synchronous activity in the low glial culture. Thus, the functional structure detected by the FCA reflects the expected changes due to the impact of the glial network on the neuronal network as discussed above.

We also quantified the amount of synchronization present within the functional clusters by comparing the scaled significance used in the joining steps of the FCA. Cultures from the HGG showed greater values of significance indicating that the firing events within the bursts are more highly synchronized. The fact that these cultures have a higher 
glial density indicates that the glial network aids in the synchronization of the neuronal network, which is again consistent with what we expected from structural observations. The significance used in clustering increased as a function of DIV in both groups indicating that as the neuronal networks evolve, the firing events become increasingly correlated. Thus, the overall observed synchronization of bursts is the result of changes in both the neuronal network and the underlying glial network.

Despite the fact that dissociated neuronal cultures are a simplified system and their structure and dynamics cannot be directly linked to brain dynamics, we have shown that they are a good reduced system in which to study the interplay of structure and dynamics in neuronal networks. Unlike neuronal data recorded from the intact brain of humans or animals where it is difficult to study the properties of the underlying network structure, we are able to manipulate gross properties of the anatomical network structure and observe how these changes affect neuronal dynamics. We can then apply methods developed to detect functional structure (such as the FCA) and relate the differences in the obtained clusterings to the known structural changes. Understanding the interaction between structural and functional network properties will be essential in advancing the study of neuronal networks both in reduced and in more complex systems. The implementation of novel analysis methods, such as the FCA, allow for the discovery of the important parameters that affect the relationships between structure and dynamics. Although we apply this analysis to a reduced system in this paper, these discoveries can later be used to make inferences about network properties that can direct research in more complicated systems such as the brain.

\section{Acknowledgments}

This work was funded through the NSF (SF), NIH grant EB008163 (MZ) and the Molecular Biophysics Training Program at the University of Michigan (JW). The authors would also like to thank the Center for the Study of Complex Systems and the Center for Advanced Computing at the University of Michigan for the use of their computing resources to analyze data in this paper. Additionally, they thank the Microscopy and Image Analysis Laboratory (MIL) at the University of Michigan, Department of Cell and Developmental Biology with the assistance of Chris Edwards for the use of their microscopes.

\section{References}

[1] Newman M E J 2006 Modularity and community structure in networks Proc. Natl Acad. Sci. USA 103 8577-82

[2] Watts D J and Strogatz S H 1998 Collective dynamics of 'small-world' networks Nature 393 440-2

[3] Guimera R, Mossa S, Turtschi A and Amaral L A 2005 The worldwide air transportation network: anomalous centrality, community structure, and cities' global roles Proc. Natl Acad. Sci. USA 102 7794-9

[4] Leicht E A and Newman M E J 2008 Community structure in directed networks Phys. Rev. Lett. 100118703
[5] Barabasi A L, Albert R and Jeong H 2000 Scale-free characteristics of random networks: the topology of the world-wide web Physica A 281 69-77

[6] Eldawlatly S, Jin R and Oweiss K G 2008 Identifying functional connectivity in large-scale neural ensemble recordings: a multiscale data mining approach Neural Comput. 21 450-77

[7] Fingelkurts A A, Fingelkurts A A and Kahkonen S 2005 Functional connectivity in the brain-is it an elusive concept? Neurosci. Biobehav. Rev. 28 827-36

[8] Friston K J, Frith C D, Liddle P F and Frackowiak R S J 1993 Functional connectivity: the principal-component analysis of large (PET) data sets J. Cereb. Blood Flow Metab. $135-14$

[9] Zhou C S, Zemanova L, Zamora-Lopez G, Hilgetag C C and Kurths J 2007 Structure-function relationship in complex brain networks expressed by hierarchical synchronization New J. Phys. 9178

[10] Honey C J, Kotter R, Breakspear M and Sporns O 2007 Network structure of cerebral cortex shapes functional connectivity on multiple time scales Proc. Natl Acad. Sci. USA 104 10240-5

[11] Honey C J, Sporns O, Cammoun L, Gigandet X, Thiran J P, Meuli R and Hagmann P 2009 Predicting human resting-state functional connectivity from structural connectivity Proc. Natl Acad. Sci. USA 106 2035-40

[12] Bowman F D, Derado G and Chen S 2009 Evaluating functional connectivity using fMRI data with diffusion-based anatomical weighting NeuroImage 47 S147

[13] Koch M A, Norris D G and Hund-Georgiadis M 2002 An investigation of functional and anatomical connectivity using magnetic resonance imaging NeuroImage 16 241-50

[14] Bogaard A, Parent J, Zochowski M and Booth V 2009 Interaction of cellular and network mechanisms in spatiotemporal pattern formation in neuronal networks J. Neurosci. 29 1677-87

[15] Feldt S, Osterhage H, Mormann F, Lehnertz K and Zochowski M 2007 Internetwork and intranetwork communications during bursting dynamics: applications to seizure prediction Phys. Rev. E 76021920

[16] Netoff T I, Clewley R, Arno S, Keck T and White J A 2004 Epilepsy in small-world networks J. Neurosci. 24 8075-83

[17] Feldt S, Waddell J, Hetrick V L, Berke J D and Zochowski M 2009 A functional clustering algorithm for the analysis of dynamic network data Phys. Rev. E 79056104

[18] Dichter M A 1978 Rat cortical neurons in cell culture: culture methods, cell morphology, electrophysiology, and synapse formation Brain Res. 149 279-93

[19] Potter S M and DeMarse T B 2001 A new approach to neural cell culture for long-term studies J. Neurosci. Methods 110 17-24

[20] Baruchi I and Ben-Jacob E 2007 Towards neuro-memory-chip: imprinting multiple memories in cultured neural networks Phys. Rev. E 75050901

[21] Shahaf G and Marom S 2001 Learning in networks of cortical neurons J. Neurosci. 21 8782-8

[22] Segev R, Benveniste M, Shapira Y and Ben-Jacob E 2003 Formation of electrically active clusterized neural networks Phys. Rev. Lett. 90168101

[23] Cohen E, Ivenshitz M, Amor-Baroukh V, Greenberger V and Segal M 2008 Determinants of spontaneous activity in networks of cultured hippocampus Brain Res. 1235 21-30

[24] Wagenaar D A, Pine J and Potter S M 2006 An extremely rich repertoire of bursting patterns during the development of cortical cultures BMC Neurosci. 711

[25] Rolston J D, Wagenaar D A and Potter S M 2007 Precisely timed spatiotemporal patterns of neural activity in dissociated cortical cultures Neuroscience 148 294-303 
[26] Baruchi I, Volman V, Raichman N, Shein M and Ben-Jacob E 2008 The emergence and properties of mutual synchronization in in vitro coupled cortical networks Eur. J. Neurosci. 28 1825-35

[27] Ham M I, Bettencourt L M, McDaniel F D and Gross G W 2008 Spontaneous coordinated activity in cultured networks: analysis of multiple ignition sites, primary circuits, and burst phase delay distributions J. Comput. Neurosci. 24 346-57

[28] Raichman N and Ben-Jacob E 2008 Identifying repeating motifs in the activation of synchronized bursts in cultured neuronal networks J. Neurosci. Methods 170 96-110

[29] Pasquale V, Massobrio P, Bologna L L, Chiappalone M and Martinoia S 2008 Self-organization and neuronal avalanches in networks of dissociated cortical neurons Neuroscience 153 1354-69

[30] Ivenshitz M and Segal M 2006 Simultaneous NMDA-dependent long-term potentiation of EPSCs and long-term depression of IPSCs in cultured rat hippocampal neurons J. Neurosci. 26 1199-210

[31] Li Y, Zhou W, Li X, Zeng S and Luo Q 2007 Dynamics of learning in cultured neuronal networks with antagonists of glutamate receptors Biophys. J. 93 4151-8

[32] Sombati S and Delorenzo R J 1995 Recurrent spontaneous seizure activity in hippocampal neuronal networks in culture J. Neurophysiol. 73 1706-11

[33] Srinivas K V, Jain R, Saurav S and Sikdar S K 2007 Small-world network topology of hippocampal neuronal network is lost, in an in vitro glutamate injury model of epilepsy Eur. J. Neurosci. 25 3276-86

[34] Furshpan E J and Potter D D 1989 Seizure-like activity and cellular damage in rat hippocampal neurons in cell culture Neuron 3 199-207

[35] Wallace T L and Johnson E M Jr 1989 Cytosine arabinoside kills postmitotic neurons: evidence that deoxycytidine may have a role in neuronal survival that is independent of dna synthesis J. Neurosci. 9 115-24

[36] Brewer G J, Torricelli J R, Evege E K and Price P J 1993 Optimized survival of hippocampal neurons in B27-supplemented Neurobasal, a new serum-free medium combination J. Neurosci. Res. 35 567-76

[37] Baltz T, de Lima A D and Voigt T 2010 Contribution of gabaergic interneurons to the development of spontaneous activity patterns in cultured neocortical networks Front Cell Neurosci. 415

[38] Fellin T 2009 Communication between neurons and astrocytes: relevance to the modulation of synaptic and network activity $J$. Neurochem. 108 533-44
[39] Pfrieger F W and Barres B A 1997 Synaptic efficacy enhanced by glial cells in vitro Science 277 1684-7

[40] Nett W J, Oloff S H and McCarthy K D 2002 Hippocampal astrocytes in situ exhibit calcium oscillations that occur independent of neuronal activity $J$. Neurophysiol. 87 528-37

[41] Pasti L, Volterra A, Pozzan T and Carmignoto G 1997 Intracellular calcium oscillations in astrocytes: a highly plastic, bidirectional form of communication between neurons and astrocytes in situ J. Neurosci. 17 7817-30

[42] Verderio C, Bacci A, Coco S, Pravettoni E, Fumagalli G and Matteoli M 1999 Astrocytes are required for the oscillatory activity in cultured hippocampal neurons Eur. $J$. Neurosci. 11 2793-800

[43] Volman V, Gerkin R C, Lau P M, Ben-Jacob E and Bi G Q 2007 Calcium and synaptic dynamics underlying reverberatory activity in neuronal networks Phys. Biol. 4 91-103

[44] Takayama Y and Moriguchi H 2009 Spontaneous calcium transients in cultured cortical networks during development IEEE Trans. Biomed. Eng. 56 2949-56

[45] Fellin T, Halassa M M, Terunuma M, Succol F, Takano H, Frank M, Moss S J and Haydon P G 2009 Endogenous non-neuronal modulators of synaptic transmission control cortical slow oscillations in vivo Proc. Natl Acad. Sci. USA 106 15037-42

[46] Sholl D A 1953 Dendritic organization in the neurons of the visual and motor cortices of the cat J. Anat. $87387-406$

[47] Date A, Bienenstock E and Geman S 1998 On the temporal resolution of neural activity Technical Report Division of Applied Mathematics, Brown University

[48] Soussou W V, Yoon G J, Brinton R D and Berger T W 2007 Neuronal network morphology and electrophysiology of hippocampal neurons cultured on surface-treated multielectrode arrays IEEE Trans. Biomed. Eng. 54 1309-20

[49] Chang J C, Brewer G J and Wheeler B C 2006 Neuronal network structuring induces greater neuronal activity through enhanced astroglial development J. Neural Eng. 3 217-26

[50] Barahona M and Pecora L M 2002 Synchronization in small-world systems Phys. Rev. Lett. 89054101

[51] Hong H, Choi M Y and Kim B J 2002 Synchronization on small-world networks Phys. Rev. E 65026139

[52] Wang X F and Chen G R 2002 Synchronization in small-world dynamical networks Int. J. Bifurcation Chaos 12 187-92 